\title{
The bargaining set and the kernel for almost-convex games
}

\author{
Jesús Getán · Josep M Izquierdo · Jesús Montes ·
}

Carles Rafels

the date of receipt and acceptance should be inserted later

\begin{abstract}
We extend well-known results for convex games (Maschler et al., 1972) to a more general class of games named almost-convex games, that is, games where all proper subgames are convex. We prove that for any balanced almost-convex game the bargaining set coincides with the core. We also prove that the kernel of any zero-monotonic almost-convex game reduces to the nucleolus. In contrast to the above results, the core of a balanced almostconvex game is stable in the sense of von Neumann-Morgenstern if and only if the game is convex.
\end{abstract}

Keywords Bargaining set $\cdot \mathrm{kernel} \cdot$ almost-convex games $\cdot$ convex games

Jesús Getán, Josep M Izquierdo, Jesús Montes, Carles Rafels

Department of Actuarial, Financial and Economic Mathematics,

University of Barcelona, Avd. Diagonal, 690; E-08034 Barcelona, Spain.

E-mail: jesus_getan@ub.edu,jesus.montes@ub.edu,jizquierdoa@ub.edu,crafels@ub.edu

Jesús Montes

Department of Economics and Bussines

Abat Oliba CEU University, Bellesguard, 30; E-08022 Barcelona, Spain.

E-mail: montes3@uao.es 


\section{Introduction and preliminaries}

Convex cooperative games (Shapley, 1971) play a central role in the development and analysis of the cooperative game theory, both in the aspect of theoretical analysis (see Csóka et al. (2011), Branzei et al.(2009) or Pulido and Sánchez-Soriano (2009) among others) and in applications of operations research (see Borm et al.(2002), Hamers (1997) or Topkis (1987)). They have a broad number of significant properties: they are totally balanced, the extreme core allocations are the marginal worth vectors, the bargaining set coincides with the core, the kernel reduces to the nucleolus, the core is the unique stable set or von Neumann-Morgenstern solution,....

In this paper we study whether the coincidence of the bargaining set with the core, the coincidence of the kernel with the nucleolus and the stability of the core still hold if we decrease the worth of the grand coalition of a convex game beyond the threshold where convexity still holds. Therefore, this paper is a sensitivity analysis of these three well-known properties when shifting the worth of the grand coalition of a convex game. A similar analysis for the extreme core allocations is done in Nuñez and Rafels (1998) and a first theoretical characterization of the coincidence between the bargaining set and the core and the inclusion of the kernel in the core for superadditive games and $\mathrm{N}$-zero-monotonic games is done in Solymosi (1999).

A cooperative game with transferable utility is a pair $(N, v)$, where $N=\{1,2, \ldots, n\}$ is the set of players and $v$ is the characteristic function, $v: 2^{N} \rightarrow \mathbb{R}$, assigning to every coalition $S \subseteq N$ a real number $v(S)$, the worth of $S$, with $v(\varnothing)=0$. We denote by $\mathscr{G}^{N}$ the set of all games on $N=\{1, \ldots, n\}$. Given a nonempty coalition $S \subseteq N$, we denote by $\left(S, v_{S}\right)$ (or $(S, v)$ if no confusion arises) the subgame of $(N, v)$ related to coalition $S$ (i.e. $v_{S}(R)=v(R)$ for all $R \subseteq S$ ). A game $(N, v)$ is monotonic if $v(S) \leq v(T)$, for all $S \subseteq T \subseteq N$. Moreover, it is zero- 
monotonic if $v(S)+\sum_{i \in T \backslash S} v(\{i\}) \leq v(T)$, for all $S \subseteq T \subseteq N$. A game $(N, v)$ is superadditive if $v(S)+v(T) \leq v(S \cup T)$ for all $S, T \subseteq N$ with $S \cap T=\varnothing$

A payoff allocation is a vector $z=\left(z_{i}\right)_{i \in N} \in \mathbb{R}^{N}$, where $z_{i}$ is the payoff to player $i \in N$. We write $z(S)=\sum_{i \in S} z_{i}$ for any nonempty coalition $S \subseteq N$ and $z(\varnothing)=0$. A payoff allocation $z \in \mathbb{R}^{N}$ is a preimputation of a game $(N, v)$ when $z(N)=v(N)$ (efficiency). The set of all the preimputations of a game $v$ is denoted by $I^{*}(N, v)$. Moreover, we say that a preimputation $z \in I^{*}(N, v)$ is an imputation of the game $(N, v)$ when $z_{i} \geq v(\{i\})$ for all $i \in N$ (individual rationality). The set of all the imputations of a game $v$ is denoted by $I(N, v)$. A game $(N, v)$ is said to be essential if $I(N, v) \neq \varnothing$ (i.e., if $\left.\sum_{i \in N} v(\{i\}) \leq v(N)\right)$. The core of a game $(N, v)$ is the set $C(N, v)=\{z \in I(N, v) \mid z(S) \geq v(S)$ for all $S \subseteq N\}$. The nonemptiness of the core can be characterized by using balanced collections of coalitions.

A nonempty collection $\mathscr{C} \subseteq 2^{N}$ is called balanced (over $N$ ) if for all $S \in \mathscr{C}$ a positive number $\delta_{S}>0$ exists such that $\sum_{S \in \mathscr{C}} \delta_{S} \chi^{S}=\chi^{N}$, where $\chi^{S} \in \mathbb{R}^{N}$ is the characteristic vector of $S$ given by $\chi_{i}^{S}=1$ if $i \in S$, and $\chi_{i}^{S}=0$ if $i \in N \backslash S$. The collection $\left(\delta_{S}\right)_{S \in \mathscr{C}}$ is called a balancing weights system. A game $(N, v)$ is said to be balanced if for each balanced collection $\mathscr{C}$ with weights $\left(\delta_{S}\right)_{S \in \mathscr{C}}$

$$
\sum_{S \in \mathscr{C}} \delta_{S} v(S) \leq v(N)
$$

We denote by $\mathscr{B}_{N}$ the set of all balanced collection of $N$. Bondareva (1963) and Shapley(1967) proved independently that a necessary and sufficient condition for the nonemptiness of the core of a game $(N, v)$ is its balancedness. In fact, in order to guarantee a game to be balanced, it is enough to evaluate condition (1) for minimal balanced collections (with respect to the inclusion). A game $(N, v)$ is said to be totally balanced if the subgames $\left(S, v_{S}\right)$ are balanced for any nonempty coalition $S \subseteq N$. 
The marginal contribution of a player $i \in N$ to a coalition $S \subseteq N \backslash\{i\}$ in the game $(N, v)$ is $v(S \cup\{i\})-v(S)$. The convexity of a cooperative game (Shapley, 1971) is defined by means of increasing marginal contributions of players whenever coalitions where players add becomes larger (snowballing effect). We said a game $(N, v)$ is convex if for all $i \in N$ and for all $S \subseteq T \subseteq N \backslash\{i\}$

$$
v(S \cup\{i\})-v(S) \leq v(T \cup\{i\})-v(T) .
$$

It is well-known that convex games are totally balanced. The proof is done by checking that marginal worth vectors associated to the different ordering of players are all core elements.

An ordering on the player set $N$ is a bijection $\theta:\{1,2, \ldots, n\} \rightarrow N$. We denote an arbitrary ordering by $\theta=\left(i_{1}, i_{2}, \ldots, i_{n}\right)$, where $i_{k}=\theta(k) \in N$, and by $\Theta_{N}$ the set of all such orderings. Given a game $v \in \mathscr{G}^{N}$ and an ordering $\theta=\left(i_{1}, i_{2}, \ldots, i_{n}\right)$, we define the marginal worth vector $m^{\theta}(v)=\left(m_{i}^{\theta}\right)_{i \in N} \in \mathbb{R}^{N}$ associated to $v$ and $\theta$ as:

$$
\begin{aligned}
& m_{i_{1}}^{\theta}=v\left(\left\{i_{1}\right\}\right), \\
& m_{i_{k}}^{\theta}=v\left(\left\{i_{1}, i_{2}, \ldots, i_{k}\right\}\right)-v\left(\left\{i_{1}, i_{2}, \ldots, i_{k-1}\right\}\right), \text { for all } k=2, \ldots, n
\end{aligned}
$$

It is well-known that a game $(N, v)$ is zero-monotonic if and only if all marginal worth vectors are imputations, $m^{\theta}(v) \in I(N, v)$, and that the game is convex if and only if all of them are core elements, $m^{\theta}(v) \in C(N, v)$ (Shapley, 1971; Ichiishi, 1981). An equivalent definition of convexity is the supermodularity of its characteristic function,

$$
v(S)+v(T) \leq v(S \cup T)+v(S \cap T), \text { for all } S, T \subseteq N .
$$

A game $(N, v)$ is almost-convex if condition (2) holds for all $S, T \subseteq N$ such that $S \cup T \neq$ $N$. That is equivalent to say that all proper subgames $\left(S, v_{S}\right), S \neq \varnothing, N$, are convex. Therefore, the class of almost-convex games includes the class of convex games and they also form a 
convex cone in $\mathscr{G}^{N}$. Moreover, balanced almost-convex games are totally balanced games and so superadditive and zero-monotonic.

Let $(N, v)$ be an essential game, let $x \in I(N, v)$ and let $i, j \in N$ be two different players. A pair $(S, y)$ is an objection at $x$ of player $i$ against $j$ when

$$
i \in S \subseteq N \backslash\{j\}, y \in \mathbb{R}^{S}, y(S)=v(S) \text { and } y_{k}>x_{k} \text { for all } k \in S .
$$

A counter-objection to the above objection $(S, y)$ is a pair $(T, z)$ where

$$
\begin{aligned}
& j \in T \subseteq N \backslash\{i\}, z \in \mathbb{R}^{T}, z(T)=v(T), \\
& z_{k} \geq y_{k} \text { for all } k \in T \cap S, \text { and } z_{k} \geq x_{k} \text { for all } k \in T \backslash S .
\end{aligned}
$$

An objection is justified if there is no counter-objection to it. The bargaining set of a game $(N, v)$ (Davis and Maschler, 1963, 1967) is the set

$$
\mathscr{M}_{1}^{i}(N, v)=\{x \in I(N, v) \mid \text { there is no justified objection at } x\} .
$$

By definition, we have $C(N, v) \subseteq \mathscr{M}_{1}^{i}(N, v) \subseteq I(N, v)$ since each core allocation cannot be objected. Moreover, $\mathscr{M}_{1}^{i}(N, v)$ is always nonempty provided the game $(N, v)$ is essential (Davis and Maschler, 1963).

A nonempty subset of the bargaining set of a game is the kernel (Davis and Maschler, 1965). Let $(N, v)$ be a game and $x \in I(N, v)$. The maximun surplus of player $i \in N$ over player $j \in N$ with respect to the imputation $x$ in the game $(N, v)$ is given by $s_{i j}^{v}(x)=\max \{v(S)-$ $\left.x(S) \mid S \in \Gamma_{i j}\right\}$, where $\Gamma_{i j}=\{S \subset N \mid i \in S, j \notin S\}$ is the set of all coalitions containing player $i$ but not player $j$. The kernel $^{1}$ of a zero-monotonic game $(N, v), \mathscr{K}(N, v)$ can be defined as the set of all imputations $x \in I(N, v)$ satisfying $s_{i j}^{v}(x)=s_{j i}^{v}(x)$ for all $i, j \in N, i \neq j$.

\footnotetext{
${ }^{1}$ In fact, this definition corresponds to the set of imputations contained in the prekernel of the game. However, we adopt this definition since the kernel coincides with the prekernel for zero-monotonic games (see Mashler et al, 1979).
} 
Maschler et al. (1972) show that the bargaining set of a convex game coincides with its core and its kernel reduces to the nucleolus of the game, defined as the unique imputation of the game that lexicographically minimizes the vector of non-increasingly ordered excesses over the set of imputations (Schmeidler, 1969). We denote by $\eta(v)$ the nucleolus of the game $(N, v)$. Let us point out that the nucleolus always belongs to the kernel of any essential game.

In Section 2 we prove the main result of the paper, that is the Core Equivalence Theo$\mathrm{rem}^{2}$ for balanced almost-convex games. The analysis of the kernel, the nucleolus and the stability of the core constitute Section 3 .

\section{The bargaining set}

To prove the core and the bargaining set of a balanced almost-convex game do coincide, we prove that any imputation not in the core of the game is not in its bargaining set. To this aim let us introduce the basic concepts of excess of a coalition and excess game and stress some important properties related to balanced almost-convex games.

Given a game $(N, v)$ and an imputation $x \in I(N, v)$, the excess of a coalition $S \subseteq N$ at $x$ is $e_{v, x}(S)=v(S)-x(S)$. The excess game of $v$ at $x$ is the game $\left(N, e_{v, x}\right)$ that assigns to each coalition $S \subseteq N$ its corresponding excess at $x$. Notice $e_{v, x}(N)=0$ and $e_{v, x}(\{i\}) \leq 0$ for all $i \in N$. Moreover, the excess game $\left(N, e_{v, x}\right)$ is balanced whenever the initial game $(N, v)$ is balanced. Finally, it is easy to check that the excess game $\left(N, e_{v, x}\right)$ is almost-convex if $(N, v)$ is almost-convex, for any $x \in I(N, v)$. The maximal excess game of $v$ at $x$, denoted by

${ }^{2}$ We use "Core Equivalence Theorem" as synonymous of the coincidence of the bargaining set of a game with its core. 
$\left(N, \hat{e}_{v, x}\right)$, is the monotonic cover of the excess game of $v$ at $x$, that is, for all $S \subseteq N$

$$
\hat{e}_{v, x}(S)=\max _{R \subseteq S} e_{v, x}(R)
$$

Notice $\hat{e}_{v, x}(N)=0$, if $x \in C(N, v)$ and vice versa. Moreover $\hat{e}_{v, x}(N)$ represents the largest excess of $v$ at $x$. Given $x \in I(N, v)$, we denote by $M(v, x)$ the set of coalitions with largest excess of $v$ at $x$, that is

$$
M(v, x)=\left\{S \subseteq N \mid e_{v, x}(S)=\hat{e}_{v, x}(N)\right\}
$$

Notice $M(v, x) \neq \varnothing$ for any game $(N, v)$ and $x \in I(N, v)$. Moreover, $M(v, x)$ is a finite ordered set with respect to the inclusion and $\varnothing \notin M(v, x)$ if $x \in I(N, v) \backslash C(N, v)$.

Given a balanced almost-convex game and an imputation outside the core of the game, let us first check that the intersection of all coalitions with the largest excess is always a nonempty set.

Lemma 1 Let $(N, v)$ be a balanced almost-convex game with $n \geq 3$ and let $x \in I(N, v) \backslash$ $C(N, v)$, then

$$
\bigcap_{S \in M(v, x)} S \neq \varnothing
$$

Proof: We denote by $M_{*}(v, x)$ the set of minimal coalitions of $M(v, x)$ w.r.t. the inclusion. It is straightforward that $\bigcap_{S \in M(v, x)} S=\bigcap_{S \in M_{*}(v, x)} S$. If $\left|M_{*}(v, x)\right|=1$, say $M_{*}(v, x)=\{R\}$ then $R \neq \varnothing$ since $x \notin C(N, v)$. Therefore $\bigcap_{S \in M(v, x)} S=R \neq \varnothing$. Let us suppose now that $\left|M_{*}(v, x)\right| \geq 2$.

We claim $S \cup S^{\prime}=N$ for any $S, S^{\prime} \in M_{*}(v, x)$, with $S \neq S^{\prime}$. If not, since $\left(N, \hat{e}_{v, x}\right)$ is an almost-convex game, we have

$$
\hat{e}_{v, x}(S)+\hat{e}_{v, x}\left(S^{\prime}\right) \leq \hat{e}_{v, x}\left(S \cup S^{\prime}\right)+\hat{e}_{v, x}\left(S \cap S^{\prime}\right) \leq \hat{e}_{v, x}(S)+\hat{e}_{v, x}\left(S \cap S^{\prime}\right)
$$

which implies $\hat{e}_{v, x}\left(S^{\prime}\right)=\hat{e}_{v, x}\left(S \cap S^{\prime}\right)$ involving a contradiction with $S^{\prime} \in M_{*}(v, x)$. 
Let $S_{*}=\bigcap_{r=1}^{k} S_{k}$ where $M_{*}(v, x)=\left\{S_{1}, \ldots, S_{k}\right\}, k \geq 2$. Assume $S_{*}=\varnothing$ then, by the above claim, $Q_{r}=N \backslash S_{r}, r=1, \ldots, k$ forms a partition of $N$. Thus $\left\{S_{1}, \ldots, S_{k}\right\}$ forms a balanced collection with weights $\delta_{S_{r}}=\frac{1}{k-1}$ for all $r=1, \ldots, k$. Since the game $(N, v)$ is balanced, then $\left(N, e_{v, x}\right)$ is also a balanced game, and thus

$$
0<\sum_{r=1}^{k} e_{v, x}\left(S_{r}\right) \leq(k-1) \cdot e_{v, x}(N)=0
$$

reaching a contradiction. Therefore, $S_{*}=\bigcap_{S \in M_{*}(v, x)} S=\bigcap_{S \in M(v, x)} S \neq \varnothing$.

Using the previous result we show the main theorem of the paper.

Theorem 1 Let $(N, v)$ be a balanced almost-convex game. Then

$$
\mathscr{M}_{1}^{i}(N, v)=C(N, v) .
$$

PROOF: For $1 \leq n \leq 2$ the proof is straightforward. From now on, we suppose $n \geq 3$. Clearly $C(N, v) \subseteq \mathscr{M}_{1}^{i}(N, v)$. Let us prove that if $x \in I(N, v) \backslash C(N, v)$ then $x \notin \mathscr{M}_{1}^{i}(N, v)$. Let $S^{*} \in M(v, x)$ be a maximal coalition w.r.t the inclusion of the largest excess at $x$. We know $1<\left|S^{*}\right|<n$. By Lemma 1 we can choose $i_{0} \in \bigcap_{S \in M(v, x)} S$ and $j_{0} \in N \backslash S^{*}$. Let us point out that player $i_{0}$ belongs to $S^{*}$, since $\bigcap_{S \in M(v, x)} S \subseteq S^{*}$.

At this point let us introduce the game $\left(S^{*} \backslash\left\{i_{0}\right\}, w\right)$ defined as follows:

$$
\begin{aligned}
& w(S):=\max _{j_{0} \in Q \subseteq N \backslash S^{*}}\left\{e_{v, x}(S \cup Q)\right\}, \text { for all } \varnothing \neq S \subseteq S^{*} \backslash\left\{i_{0}\right\}, \\
& w(\varnothing):=0 .
\end{aligned}
$$

We claim $\left(S^{*} \backslash\left\{i_{0}\right\}, w\right)$ is a convex game. To see it, let $S, T \subseteq S^{*} \backslash\left\{i_{0}\right\}$ be two arbitrary coalitions and let $Q_{S}, Q_{T} \subseteq N \backslash S^{*}$ be the corresponding coalitions satisfying $w(S)=e_{v, x}(S \cup$ $\left.Q_{S}\right)$ and $w(T)=e_{v, x}\left(T \cup Q_{T}\right)$. Notice we have $(S \cup T) \cup\left(Q_{S} \cup Q_{T}\right) \subseteq N \backslash\left\{i_{0}\right\}$, which implies $(S \cup T) \cup\left(Q_{S} \cup Q_{T}\right) \neq N$ and $\left(S \cup Q_{S}\right) \cap\left(T \cup Q_{T}\right)=(S \cap T) \cup\left(Q_{S} \cap Q_{T}\right)$, since $S \cap Q_{T}=\varnothing$ 
and $T \cap Q_{S}=\varnothing$. Hence, by the almost-convexity of the game $\left(N, e_{v, x}\right)$ we obtain

$$
\begin{aligned}
w(S)+w(T) & =e_{v, x}\left(S \cup Q_{S}\right)+e_{v, x}\left(T \cup Q_{T}\right) \\
& \leq e_{v, x}\left(S \cup T \cup Q_{S} \cup Q_{T}\right)+e_{v, x}\left((S \cap T) \cup\left(Q_{S} \cap Q_{T}\right)\right) \\
& \leq w(S \cup T)+w(S \cap T),
\end{aligned}
$$

proving our claim, $\left(S^{*} \backslash\left\{i_{0}\right\}, w\right)$ is a convex game. Now consider the corresponding monotonic cover $\left(S^{*} \backslash\left\{i_{0}\right\}, \hat{w}\right)$ defined by

$$
\begin{aligned}
\hat{w}(S) & =\max _{R \subseteq S} w(R)=\max \left\{0, \max _{\varnothing \neq R \subseteq S}\{w(R)\}\right\} \\
& =\max \left\{0, \max _{\varnothing \neq R \subseteq S}\left\{\max _{j_{0} \in Q \subseteq N \backslash S^{*}}\left\{e_{v, x}(R \cup Q)\right\}\right\}, \text { for all } S \subseteq S^{*} \backslash\left\{i_{0}\right\} .\right.
\end{aligned}
$$

Since $\left(S^{*} \backslash\left\{i_{0}\right\}, \hat{w}\right)$ is the monotonic cover of the convex game $\left(S^{*} \backslash\left\{i_{0}\right\}, w\right)$ it is also a convex game. We claim $\Delta:=\hat{e}_{v, x}(N)-\hat{w}\left(S^{*} \backslash\left\{i_{0}\right\}\right)>0$. To see it, if $\hat{w}\left(S^{*} \backslash\left\{i_{0}\right\}\right)=0$ we get $\Delta>0$ since $\hat{e}_{v, x}(N)>0$, for any $x \in I(N, v) \backslash C(N, v)$. Otherwise, $\hat{w}\left(S^{*} \backslash\left\{i_{0}\right\}\right)=e_{v, x}(R \cup Q)$ for some $\varnothing \neq R \subseteq S^{*} \backslash\left\{i_{0}\right\}$ and $j_{0} \in Q \subseteq N \backslash S^{*}$. Notice that $i_{0} \notin Q$ since $i_{0} \in S^{*}$. Therefore $\hat{w}\left(S^{*} \backslash\left\{i_{0}\right\}\right)=e_{v, x}(R \cup Q) \leq \hat{e}_{v, x}(N)$. If $e_{v, x}(R \cup Q)=\hat{e}_{v, x}(N)$ then $R \cup Q \in M(v, x)$, which involves a contradiction since $i_{0} \in \bigcap_{S \in M(v, x)} S$ but $i_{0} \notin R \cup Q$.

Being $\left(S^{*} \backslash\left\{i_{0}\right\}, \hat{w}\right)$ a convex game, let $u \in C\left(S^{*} \backslash\left\{i_{0}\right\}, \hat{w}\right)$ be an arbitrary core element. We know $u_{k} \geq \hat{w}(\{k\}) \geq 0$, for any $k \in S^{*} \backslash\left\{i_{0}\right\}$.

Then, define the objection $\left(S^{*}, y\right)$ at $x$ of player $i_{0} \in \cap_{S \in M(v, x)} S \subseteq S^{*}$ against any player $j_{0} \in N \backslash S^{*}$ as

$$
\begin{aligned}
y_{i_{0}} & :=x_{i_{0}}+\frac{\Delta}{\left|S^{*}\right|}>x_{i_{0}}, \\
y_{k}: & =x_{k}+u_{k}+\frac{\Delta}{\left|S^{*}\right|}>x_{k}, \text { for } k \in S^{*} \backslash\left\{i_{0}\right\} .
\end{aligned}
$$

Notice we have

$$
\begin{aligned}
y\left(S^{*}\right) & =x\left(S^{*}\right)+u\left(S^{*} \backslash\left\{i_{0}\right\}\right)+\Delta \\
& =x\left(S^{*}\right)+\hat{w}\left(S^{*} \backslash\left\{i_{0}\right\}\right)+\Delta \\
& =x\left(S^{*}\right)+\hat{e}_{v, x}(N)=v\left(S^{*}\right),
\end{aligned}
$$


where the last equality comes from the fact that $S^{*} \in M(v, x)$.

Let us see there is no counter-objection to the objection $\left(S^{*}, y\right)$ at $x$. To this aim, let $(T, z)$ be a arbitrary counter-objection. We have $j_{0} \in T, i_{0} \notin T$ and $e_{v, x}(T) \geq 0$. First notice $T \cap S^{*} \neq \varnothing$. If not, $T \cap S^{*}=\varnothing$ and, by the superadditivity of the game $\left(N, e_{v, x}\right)$ (recall $\left(N, e_{v, x}\right)$ is a balanced almost-convex game), we have

$$
e_{v, x}\left(S^{*}\right) \leq e_{v, x}\left(S^{*}\right)+e_{v, x}(T) \leq e_{v, x}\left(S^{*} \cup T\right)
$$

which contradicts $S^{*} \in M(v, x)$ to be a maximal coalition with respect to the inclusion of the largest excess at $x$. Moreover,

$$
\begin{aligned}
z(T) & \geq y\left(T \cap S^{*}\right)+x\left(T \backslash S^{*}\right) \\
& =x\left(T \cap S^{*}\right)+u\left(T \cap S^{*}\right)+\left|T \cap S^{*}\right| \frac{\Delta}{\left|S^{*}\right|}+x\left(T \backslash S^{*}\right) \\
& >x(T)+u\left(T \cap S^{*}\right) \geq x(T)+\hat{w}\left(T \cap S^{*}\right) \\
& \geq x(T)+v\left(\left(T \cap S^{*}\right) \cup\left(T \backslash S^{*}\right)\right)-x\left(\left(T \cap S^{*}\right) \cup\left(T \backslash S^{*}\right)\right) \\
& =x(T)+v(T)-x(T)=v(T),
\end{aligned}
$$

which contradicts $(T, z)$ to be a counter-objection to the objection $\left(S^{*}, y\right)$ at $x$. This ends the proof.

The above theorem enlarges the classes of games where the bargaining set coincides with the core: assignment games (Solymosi, 1999), average monotonic games (Izquierdo and Rafels, 2001), partitioning games (Solymosi, 2008), clan games (Potters et al., 1989), monotonic simple games with veto control (Einy and Wettstein, 1996), etc. This coincidence is known in the literature as the Core Equivalence Theorem (Einy et al., 1997) and it gives insights into the core allocations: they are the unique allocations robust in front to objections.

Moreover, in our case we can also see Theorem 1 as an extension of the corresponding result for the class of convex games. To evaluate the relevance of Theorem 1 it is important to 
know its applicability range. In other words, how different are the class of balanced almostconvex games and the class of convex games? To check this point, we introduce and recall some concepts.

A game $(N, v)$, with $n \geq 2$, is rooted if the nonemptiness of the core starts just at its efficiency level (see Calleja et al., 2009). Furthermore, to every game $(N, v)$ we can associate its unique rooted game $\left(N, v_{r}\right)$ just by moving appropriately the worth of the grand coalition, that is, $v_{r}(S)=v(S)$, for $S \neq N$ and

$$
v_{r}(N)=\max _{\substack{\mathscr{C} \in \mathscr{B}_{N}, \mathscr{C} \neq\{N\} \text { and } \\ \text { minimal w.r.t. the inclusion }}} \sum_{S \in \mathscr{C}} \delta_{S} v(S) .
$$

With this notation at hand, a game $(N, v), n \geq 2$, is rooted if it coincides with its rooted game, i.e. $v=v_{r}$. Using this concept, notice that any convex game $(N, v)$ such that its rooted game $\left(N, v_{r}\right)$ is not convex illustrates the applicability of our Theorem 1 . This represents a majority of convex games, since to be $\left(N, v_{r}\right)$ a convex game requires that the maximum in (4) is attained at a partition formed by only two nonempty subsets of $N$. This argument is stated in the the next proposition.

Proposition 1 Let $(N, v), n \geq 2$, be a convex game. The following statements are equivalent:

1. $(N, v)$ is a rooted game.

2. There exists a nonempty coalition $S, S \neq N$, such that

$$
v(N)=v(S)+v(N \backslash S) .
$$

Proof: 1. $\rightarrow$ 2.) Let $(N, v)$ be a rooted convex game, i.e. $(N, v)$ is convex and $v=v_{r}$. Since $v$ is rooted, by (4), there must exist a coalition $S \neq \varnothing, N$ satisfying $x(S)=v(S)$, for all $x \in C(N, v)$. Now, take any ordering $\theta=\left(i_{1}, \ldots, i_{n}\right)$ on $N$, where the members of coalition $S$ occupy the last positions, i.e. $S=\left\{i_{n-s+1}, \ldots, i_{n}\right\}$, where $s$ represents the cardinality of coalition $S$, and let $m^{\theta}(v)$ be the corresponding marginal worth vector of $v$ associated to 
$\theta$. Since the game $(N, v)$ is convex, we have $m^{\theta}(v) \in C(N, v)$ and so $m^{\theta}(S)=v(S)$, since $x(S)=v(S)$ for all $x \in C(N, v)$. Moreover, by the specific ordering $\theta$, we have $m^{\theta}(S)=$ $m^{\theta}(N)-m^{\theta}(N \backslash S)=v(N)-v(N \backslash S)$. Therefore, $(N, v)$ satisfies condition 2.

2. $\rightarrow$ 1.) It is straightforward since, being $(N, v)$ a convex game, the equality in condition 2 implies that the game is rooted.

There is another possibility to characterize rooted convex games in terms of its unanimity coordinates. For all nonempty coalition $T \subseteq N$, the unanimity game $\left(N, u_{T}\right)$ is defined by $u_{T}(S)=1$, if $S \supseteq T$ and $u_{T}(S)=0$, otherwise. Any game $(N, v)$ can be expressed as a linear combination of unanimity games, i.e. $v=\sum_{T \subseteq N} \lambda_{T} \cdot u_{T}$. Using these concepts we can state now that a convex game $(N, v)$, with $v=\sum_{R \subseteq N} \lambda_{R} u_{R}$, is rooted if and only if there exists $S \neq \varnothing, N$ such that $\lambda_{R}=0$ when $R \cap S \neq \varnothing$ and $R \cap(N \backslash S) \neq \varnothing$. The straightforward proof is left to the reader.

A direct application of Proposition 1 is to check that the rooted game associated to any strictly convex ${ }^{3}$ game, with $n \geq 3$, is a balanced almost-convex game but not convex ${ }^{4}$. This shows that, starting from a strictly convex game and lowering the efficiency level, we will reach some efficiency level where the game is non-convex but balanced. For all these cases, Theorem 1 applies and states a non-obvious coincidence of the core and the bargaining set.

\footnotetext{
3 A game $(N, v)$ is strictly convex if $v(S)+v(T)<v(S \cup T)+v(S \cap T)$, for any $S, T \subseteq N, S \backslash T \neq \varnothing$ and $T \backslash S \neq \varnothing$.

4 Given a strictly convex game $(N, v)$, if its rooted game $\left(N, v_{r}\right)$ is convex then we have, by Proposition $1, v_{r}(N)=v_{r}(S)+v_{r}(N \backslash S)$, for some nonempty coalition $S \neq N$. Taking $T \varsubsetneqq N, T \cap S \neq \varnothing, T \cap(N \backslash S) \neq$ $\varnothing(n \geq 3$ is needed $)$, and since $\left(N, v_{r}\right)$ is convex, we have that $v_{r}(T)+v_{r}(S) \leq v_{r}(T \cup S)+v_{r}(T \cap S)$ and $v_{r}(T \cup S)+v_{r}(N \backslash S) \leq v_{r}(N)+v_{r}\left(T \cap(N \backslash S)\right.$. Moreover, adding up this two inequalities we obtain $v_{r}(T) \leq$ $v_{r}(T \cap S)+v_{r}(T \cap(N \backslash S))$. Hence, by superadditivity of the game $\left(N, v_{r}\right)$, we conclude $v_{r}(T)=v_{r}(T \cap S)+$ $v_{r}(T \cap(N \backslash S))$ and so $v(T)=v(T \cap S)+v(T \cap(N \backslash S))$, which contradicts the game $(N, v)$ to be strictly convex.
} 
From the seminal paper by Davis and Maschler (1963), other definitions of bargaining sets have been introduced with the aim to qualify the concept of what objections and counter-objections are. Among the ones defined over the imputation set we want to quote the individual rational Mas-Colell bargaining set introduced by Vohra (1991), the reactive bargaining set (Granot, 2010) and a variation of the original Mas-Colell bargaining set given by Shimomura (1997). All these variations satisfy the Core Equivalence Theorem when we deal with convex games.

Let us briefly describe the behavior of the Core Equivalence Theorem for these bargaining sets on the class of balanced almost-convex games. With respect to the reactive bargaining set, it always includes the core and it is included in the Davis and Maschler bargaining set. Therefore, by Theorem 1, it satisfies the Core Equivalence Theorem within the class of balanced almost-convex games. With respect to the Shimomura's bargaining set, Izquierdo and Rafels (2011) recently prove that the only class of games where the core and the Mas-Colell bargaining set à la Shimomura coincide is the class of convex games. Therefore, for any proper (not convex) balanced almost-convex game, the Core Equivalence Theorem does not hold for the Mas-Colell bargaining set à la Shimomura. Unfortunately, we do not know whether the Core Equivalence Theorem is preserved for the individual rational bargaining of Vohra (1991) or even for the original Mas-Colell bargaining set (Mas-Colell, 1989), remaining both questions opened for future work.

\section{The kernel, the nucleolus and the stability of the core}

An important property of convex games is the coincidence of the kernel with the nucleolus (Schmeidler, 1969). We generalize this property to the class of zero-monotonic almostconvex games. Moreover, we also show another interesting property which is the fact that 
the nucleolus (and so the kernel) of a zero-monotonic almost-convex game coincides with the nucleolus (the kernel) of a suitable convex game associated. This property allows for the application of specific efficient algorithms to calculate the nucleolus of zero-monotonic almost-convex game (see Faigle et al.,2001; Arin and Iñarra, 1998; Kuipers, 1996).

Let $(N, v)$ be an arbitrary almost-convex game. Notice the nonemptiness of the core is not needed. For any $\varepsilon \geq 0$ we take the $\varepsilon$-shifted associated game $\left(N, v_{\varepsilon}\right)$ defined as $v_{\varepsilon}(S)=$ $v(S)-\varepsilon$, for all $S \neq \varnothing, N, v_{\varepsilon}(N)=v(N)$ and $v_{\varepsilon}(\varnothing)=0$.

Let $\varepsilon^{*}=\min \left\{\varepsilon \geq 0 \mid\left(N, v_{\varepsilon}\right)\right.$ is a convex game $\}$. Notice $\varepsilon^{*}$ is well-defined since $(N, v)$ is an almost-convex game. Indeed, to provide a formula for such $\varepsilon^{*}$ let us remark that analyzing the marginal contributions of an arbitrary player $i \in N$ to a coalition $\varnothing \neq S \subseteq N \backslash\{i\}, S \neq$ $N \backslash\{i\}$ in the games $(N, v)$ and $\left(N, v_{\varepsilon}\right)$ we obtain for any $\varepsilon \geq 0$

$$
\begin{aligned}
& v_{\varepsilon}(\{i\}) \leq v(\{i\}), \\
& v_{\varepsilon}(S \cup\{i\})-v_{\varepsilon}(S)=v(S \cup\{i\})-v(S) \text { and } \\
& v(N)-v(N \backslash\{i\}) \leq v_{\varepsilon}(N)-v_{\varepsilon}(N \backslash\{i\})=v(N)-v(N \backslash\{i\})+\varepsilon .
\end{aligned}
$$

Furthermore, if $(N, v)$ is an almost-convex game and we impose the corresponding $\varepsilon$ shifted game $\left(N, v_{\varepsilon}\right)$, with $\varepsilon \geq 0$, to be a convex game we obtain

$$
\varepsilon \geq \varepsilon^{*}=\max _{i \neq j}\{v(N \backslash\{i\})+v(N \backslash\{j\})-v(N)-v(N \backslash\{i, j\}), 0\},
$$

since we only have to guarantee the inequalities $v(N \backslash\{j\})-v(N \backslash\{i, j\}) \leq v(N)-v(N \backslash$ $\{i\})+\varepsilon$, for all $i, j \in N, j \neq i$. Notice $\varepsilon^{*}=0$ if the original game $(N, v)$ is convex and $\varepsilon^{*}>0$ when $(N, v)$ is a proper (not convex) almost-convex game. Next we prove our second main result. 
Theorem 2 Let $(N, v)$ be a zero-monotonic almost-convex game ${ }^{5}, n \geq 2$. Then, the kernel reduces to the nucleolus, $\mathscr{K}(N, v)=\{\eta(v)\}$. Moreover, the nucleolus of the game $(N, v)$ coincides with the nucleolus of the convex game $\left(N, v_{\varepsilon^{*}}\right)$, where $\varepsilon^{*}$ is defined in (5).

Proof: It is easy to see that for any efficient allocation $x \in \mathbb{R}^{N}, x(N)=v(N)=v_{\mathcal{E}^{*}}(N)$, we have $s_{i j}^{v_{\varepsilon^{*}}}(x)=s_{i j}^{v}(x)-\varepsilon^{*}$, for all $i \neq j \in N$. Being $\left(N, v_{\varepsilon^{*}}\right)$ a convex game and $(N, v)$ a zero-monotonic almost-convex game, we easily obtain $\mathscr{K}(N, v)=\mathscr{K}\left(N, v_{\varepsilon^{*}}\right)=\left\{\eta\left(v_{\mathcal{E}^{*}}\right)\right\}$ which implies $\eta(v)=\eta\left(v_{\mathcal{E}^{*}}\right)$ and finishes the proof.

Next example shows that zero-monotonicity is necessary in the statement of Theorem 2. Let $(N, v)$ be the three-player game given by $v(\{1\})=1, v(\{2\})=2, v(\{3\})=3$, $v(\{1,2\})=4, v(\{1,3\})=6, v(\{2,3\})=8$ and $v(\{1,2,3\})=7$. It can be checked that $\varepsilon^{*}=4$ and $\eta\left(v_{\mathcal{E}^{*}}\right)=\left(\frac{1}{3}, 2 \frac{1}{3}, 4 \frac{1}{3}\right) \notin I(N, v)$. Thus $\eta(v) \neq \eta\left(v_{\mathcal{E}_{*}}\right)$. Notice the game $(N, v)$ is almostconvex but not zero-monotonic since $v(\{1\})+v(\{2,3\})=9 \not \leq v(\{1,2,3\})=7$.

In this paper we have proved that moving down the worth of the grand coalition of a convex game does not affect the relationship between prominent solutions of cooperative games, namely the core versus the bargaining set and the kernel versus the nucleolus. We might erroneously conclude that all the relationships between cooperative solutions that holds for convex games are naturally extended to the class of almost-convex games. Now, we can check that the outstanding stability of the core (in the sense of von Neumann and Morgenstern, 1944) of convex games is not preserved for balanced almost-convex games. In fact, if the game is not convex, but balanced almost-convex, this property is lost as the next proposition states.

\footnotetext{
5 In some connexion with Solymosi (1999), note that zero-monotonic almost-convex games are equivalent to $N$-zero-monotonic almost-convex games, where $(N, v)$ is $N$-zero-monotonic if $v(S)+\sum_{i \in N \backslash S} v(\{i\}) \leq v(N)$, for all $S \subseteq N$.
} 
Before this, let us recall that given an essential game $(N, v)$ and two imputations $x, y \in$ $I(N, v)$, we say that $x$ dominates $y$ if there exists $S \subseteq N$ such that $x_{i}>y_{i}$, for all $i \in S$ and $x(S)=v(S)$. A set $V \subseteq I(N, v)$ is a stable set if: (a) for all $x, x^{\prime} \in V$ neither $x$ dominates $x^{\prime}$ nor vice versa (internal stability); (b) for all $y \in I(N, v) \backslash V$, there exist $x \in V$ such that $x$ dominates $y$ (external stability).

Proposition 2 Let $(N, v)$ be an essential almost-convex game. The following statements are equivalent.

1. $(N, v)$ is convex.

2. $C(N, v)$ is a stable set.

Proof: If an almost-convex game $(N, v)$ is convex, it is well-known its core is a stable set. Let us suppose now that the game $(N, v)$ is almost-convex and the core of $(N, v)$ is a stable set, but the game is not convex. Hence there exists a marginal worth vector relative to some ordering of players $\theta=\left(i_{1}, i_{2}, \ldots, i_{n}\right)$ which is not in the core of the game ${ }^{6}, m^{\theta}(v) \in$ $I(N, v) \backslash C(N, v)$. However, by hypothesis, the core is a stable set and so there is $z \in C(N, v)$ such that it dominates $m^{\theta}(v)$ via some coalition $S \subseteq N$. Since the game $v$ is almost-convex, the subgame $\left(N \backslash\left\{i_{n}\right\}, v\right)$ is convex and so $m^{\theta}(v)(S) \geq v(S)$, for all $S \subseteq N \backslash\left\{i_{n}\right\}$. Hence, $z$ dominates $m^{\theta}(v)$ through some coalition $S$ containing player $i_{n}$. Thus, $z_{i_{n}}>m_{i_{n}}^{\theta}=v(N)-$ $v\left(N \backslash\left\{i_{n}\right\}\right)$, but then we obtain $z\left(N \backslash\left\{i_{n}\right\}\right)<v\left(N \backslash\left\{i_{n}\right\}\right)$ which contradicts $z$ to be a core element.

6 The marginal worth vectors are imputations since the game $(N, v)$ is both balanced and almost-convex, which implies the zero-monotonicity of the game $(N, v)$. 


\section{Conclusions}

In this study, we analyze whether some properties of cooperative solutions applied to convex games still hold when the efficiency level is moved down and the convexity of the game is lost. We approach this situation by means of the analysis of almost-convex games, since the convexity structure of subgames remains unaltered . First, we have proved that the bargaining set of such games is equal to the corresponding core, whenever this latter is nonempty. This is important since it reflects that the core of a convex game is still robust in front of bargaining among players, even if some loose of efficiency occurs. This fact contrast with other types of games ${ }^{7}$ like symmetric games (see Meertens et al., 2007). We have also checked that another important property of convex games, that is the kernel reduces to the nucleolus, is also preserved for zero-monotonic almost-convex game. However other properties of convex games vanishes for the larger class of almost convex games like the von Neumann and Morgenstern stability of the core or the fact that the Shapley value (Shapley, 1953) of the game is always a core element. As the Shapley value is a population monotonic allocation scheme (Sprumont, 1990) for convex games, it remains open for future work the existence of population monotonic allocation scheme for any almost-convex game.

Acknowledgements Institutional support from Ministerio de Ciencia e Innovación and FEDER under grant ECO2011-22765, and Generalitat de Catalunya under grant 2009SGR0960 is acknowledged.

\footnotetext{
7 To see it, take for instance the five-player game defined in Maschler(1976) as $v(S)=\min \left\{|S \cap P|, \frac{1}{2} \mid S \cap\right.$ $Q\}$ for all $S \subseteq N=\{1,2,3,4,5\}$, where $P=\{1,2\}$ and $Q=\{3,4,5\}$. In his paper, Maschler determine that the bargaining set of this game is strictly larger than the corresponding core that consists of a unique point $\left(0,0, \frac{1}{2}, \frac{1}{2}, \frac{1}{2}\right)$. However, if we raise the efficiency level up to $v(N)=2.5$, it is not difficult to check that then the core and the bargaining set coincide.
} 


\section{References}

1. Arin, J., \& Iñarra, E. (1998). A characterization of the nucleolus for convex games. Games and Economic Behavior, 23, 12-24.

2. Bondareva, O.N. (1963). Some applications of linear programming methods to the theory of cooperative games. Problemi Kibernitiki, 10, 119-139.

3. Borm, P., Fiestras-Janeiro, G., Hamers, H., Sánchez, E., \& Voorneveld, M. (2002). On the convexity of games corresponding to sequencing situations with due dates. European Journal of Operational Research, $136,616-634$

4. Branzei, R., Tijs, S. \& Zarzuelo, J. (2009). Convex multi-choice games: Characterizations and monotonic allocation schemes. European Journal of Operational Research, 198, 571-575.

5. Calleja, P., Rafels, C., \& Tijs, S. (2009). The aggregate-monotonic core. Games and Economic Behavior, $66(2), 742-748$

6. Csóka, P., Herings, J.J., Kóczy, L.Á. \& Pintér, M. (2011). Convex and Exact games with non-transferable utility. European Journal of Operational Research, 209, 57-62.

7. Davis, M., \& Maschler, M. (1963). Existence of stable payoff configurations for cooperative games. Bulletin American Society, 69, 106-108.

8. Davis, M., \& Maschler, M. (1965). The kernel of a cooperative game. Naval Research Logistics Quarterly, $12,223-259$

9. Davis, M., \& Maschler, M. (1967). Existence of stable payoff configurations for cooperative games. In M. Shubik (Ed.), Essays in Mathematical Economics in Honor of Oskar Morgestern (pp. 39-52). Princeton, NJ: Princeton Univ. Press.

10. Einy, E., \& Wettstein D. (1996). Equivalence between bargaining sets and the core of simple games. International Journal of Game Theory, 25, 65-71.

11. Einy, E., Holzman, R., Monderer, D., \& Shitovitz, B. (1997). Core equivalence theorems for infinite convex games. Journal of Economic Theory, 76, 1-12.

12. Faigle, U., Kern, W., \& Kuipers, J. (2001). On the computation of the nucleolus of a cooperative game. International Journal of Game Theory, 30, 79-98.

13. Granot, D. (2010). The reactive bargaining set for cooperative games. International Journal of Game Theory, 1, 73-93.

14. Hamers, H. (1997). On the concavity of delivery games. European Journal of Operational Research, 99, $445-458$. 
15. Ichiishi, T. (1981). Supermodularity applications to convex games and to the greedy algorithm for LP. Journal of Economic Theory, 25, 283-286.

16. Izquierdo, J.M., \& Rafels, C. (2001). Average monotonic cooperative games. Games and Economic Behavior, 36, 174-192

17. Izquierdo, J.M., \& Rafels, C. (2011). A characterization of convex TU games by means of the MasColell bargaining set (à la Shimomura), forthcoming in International Journal of Game Theory, DOI: 10.1007/s00182-011-0291-9.

18. Kuipers, J. (1996). A polynomial time algorithm for computing the nucleolus of convex games. Report M 96-12, Maastricht University.

19. Mas-Colell, A. (1989). An equivalence theorem for a bargaining set. Journal of Mathematical Economics, 18, 129-139.

20. Maschler, M., Peleg, B., \& Shapley, S. (1972). The kernel and bargaining set for convex games. International Journal of Game Theory, 39, 163-170.

21. Maschler, M. (1976). An advantage of the bargaining set over the core. Journal of Economic Theory, 13, $184-192$.

22. Maschler, M., Peleg, B., \& Shapley, S. (1979). Geometric properties of kernel, nucleolus and related concepts. Mathematics of Operations Research, 4, 303-338.

23. Meertens, M., Pooters, J.A.M., \& Reijnierse, J.H. (2007). On bargaining sets in symmetric games. International Game Theory Review, 9, 199-213.

24. Núñez, M., \& Rafels, C. (1998). On extreme points of the core and reduced games. Annals of Operations Research, 84, 121-133.

25. Potters, J.A.M., Poos, R., Tijs, S.H., \& Muto S. (1989). Clan games. Games and Economic Behavior, 1, $275-293$.

26. Pulido, M., \& Sánchez-Soriano, J. (2009). On the core, the Weber set and convexity in games with a priori unions. European Journal of Operational Research, 193, 468-475.

27. Schmeidler, D. (1969). The nucleolus of a characteristic function game. SIAM Journal on Applied Mathematics, 17, 1163-1170.

28. Shapley, L. S. (1953). A Value for N-Person Games. In Kuhn and Tucker (Eds.), Contributions to the Theory of Games.(pp. 307-317). Princeton, NJ: Princeton Univ. Press.

29. Shapley, L. S. (1967). On balanced sets and cores. Naval Research Logistics Quarterly, 14, 453-460.

30. Shapley, L. S. (1971). Cores of convex games. International Journal of Game Theory, 1, 11-26. 
31. Shimomura, K. (1997). Quasi-cores in bargaining sets. International Journal of Game Theory, 26, 283302.

32. Sprumont, Y. (1990). Population monotonic allocation schemes for cooperative games with transferable utility. Games and Economic Behavior, 2, 378-394.

33. Solymosi, T. (1999). On the bargaining set, kernel and core of superadditive games. International Journal of Game Theory, 28, 229-240.

34. Solymosi, T. (2008). Bargaining sets and the core in partitioning games. CE Journal of Operations Research, 16, 425-440.

35. Topkis, D.M. (1987). Activity optimization games with complementarity. European Journal of Operational Research, 28, 358-368.

36. Vohra, R. (1991). An existence theorem for a bargaining set. Journal of Mathematical Economics, 20,1934.

37. von Neumann, J., \& Morgenstern, O. (1944). Theory of Games and Economic Behavior, Princeton University Press, Princeton. 